\title{
Therapeutic Surgical Management of Palpable Melanoma Groin Metastases: Superficial or Combined Superficial and Deep Groin Lymph Node Dissection
}

\author{
A. P. T. van der Ploeg, MSc ${ }^{1}$, A. C. J. van Akkooi, PhD $^{1}$, P. I. M. Schmitz, PhD ${ }^{2}$, A. N. van Geel, MD $^{1}$, \\ J. H. de Wilt, $\mathrm{PhD}^{1,3}$, A. M. M. Eggermont, $\mathrm{PhD}^{1,4}$, and $\mathrm{C}$. Verhoef, $\mathrm{PhD}^{1}$ \\ ${ }^{1}$ Department of Surgical Oncology, Erasmus University Medical Center - Daniel den Hoed Cancer Center, Rotterdam, The \\ Netherlands; ${ }^{2}$ Trials and Statistics, Erasmus University Medical Center - Daniel den Hoed Cancer Center, Rotterdam, The \\ Netherlands; ${ }^{3}$ Department of Surgery, Radboud University Nijmegen Medical Center, Nijmegen, The Netherlands; \\ ${ }^{4}$ Institut de Cancérologie Gustave Roussy, Villejuif, France
}

\begin{abstract}
Background. Management of patients with clinically detectable lymph node metastasis to the groin is by ilioinguinal or combined superficial and deep groin dissection (CGD) according to most literature, but in practice superficial groin dissection (SGD) only is still performed in some centers. The aim of this study is to evaluate the experience in CGD versus SGD patients in our center.

Methods. Between 1991 and 2009, 121 therapeutic CGD and 48 SGD were performed in 169 melanoma patients with palpable groin metastases at our institute. Median follow-up was 20 and, for survivors, 45 months.

Results. In this heterogeneous group of patients, overall (OS) and disease-free survival, local control rates, and morbidity rates were not significantly different between CGD and SGD patients. However, CGD patients had a trend towards more chronic lymphedema. Superficial lymph node ratio, the number of positive superficial lymph nodes, and the presence of deep nodes were prognostic factors for survival. CGD patients with involved deep lymph nodes (24.8\%) had estimated 5-year OS of $12 \%$ compared with $40 \%$ with no involved deep lymph nodes $(p=0.001)$. Preoperative computed tomography (CT) scan had high negative predictive value of $91 \%$ for detection of pelvic nodal involvement.
\end{abstract}

(C) The Author(s) 2011. This article is published with open access at Springerlink.com

First Received: 9 November 2010;

Published Online: 3 May 2011

C. Verhoef, PhD

e-mail: c.verhoef@erasmusmc.nl
Conclusions. This study demonstrated that survival and local control do not differ for patients with palpable groin metastases treated by CGD or SGD. Patients without pathological iliac nodes on CT might safely undergo SGD, while CGD might be reserved for patients with multiple positive nodes on SGD and/or positive deep nodes on CT scan.

Management of patients with clinically detectable lymph node metastasis to the groin consists of ilioinguinal or combined superficial and deep groin dissection (CGD) according to most literature. ${ }^{1-7}$ Some surgeons are willing to do so only when there are multiple positive nodes in the groin or when there is evidence of pelvic nodal involvement on the basis of imaging information. ${ }^{8}$ In practice, a solely inguinal or superficial groin dissection (SGD) is still performed in some cases and/or centers. The potential survival or local control benefit of extensive surgery remains controversial in the absence of randomized data.

Prognosis and outcome of patients after CGD are believed to correlate with the biology of the disease rather than with the extent of the operation. ${ }^{4,8-11}$ It is advocated that CGD should be performed when there is clinically gross involvement of the groin, when there are clinically detectable deep lymph nodes, when Cloquet's node is histologically positive or when pelvic computed tomography (CT) demonstrates pelvic lymphadenopathy. ${ }^{12}$

The aim of this study is to evaluate the experience in patients with clinically evident metastatic melanoma to the groin who underwent CGD versus SGD only. Postoperative morbidity, regional recurrence, preoperative CT scan, and disease-free and overall survival were analyzed. 
The necessity of removal of the deep iliac and obturator lymph nodes as well as prognostic factors for survival in patients with metastatic melanoma to the groin were evaluated.

\section{PATIENTS AND METHODS}

\section{Patients}

Patients in this study presented with clinically detectable metastases to the groin at the Erasmus University Medical Center - Daniel den Hoed Cancer Center, Rotterdam, The Netherlands. Patients were selected for therapeutic ilioinguinal or combined superficial and deep groin dissection (CGD) or for inguinal or superficial groin dissection (SGD). All patients underwent the operation within 2 months of detection of palpable metastasis. Patients who underwent sentinel lymph node biopsy were excluded. All patient characteristics, tumor characteristics, postoperative morbidities, regional recurrence patterns, and imaging procedures (preoperative CT scan) were collected and sorted in a database for this retrospective single-institution study. Postoperative morbidities were collected from patient charts and divided into two categories; short-term morbidities, e.g., wound infection or necrosis, and seroma, and long-term morbidities, e.g., chronic lymphedema. Chronic lymphedema was recorded if moderate or severe swelling was present for more than 6 weeks postoperatively and the patient required therapy. Adjuvant radiotherapy was given to $16(9.5 \%)$ patients, who were treated with doses between 15 and 80 Gy.

\section{Surgical Procedure}

Four coauthors performed the majority of lymph node dissections assessed for this study (J.H. de W., A.N. van G., A.M.M.E., and C.V.). In general, patients with palpable inguinal nodes underwent CGD. Indication for SGD was based on surgeon or patient preference. Patients with significant (cardiopulmonary) comorbidities and absence of preoperative radiological and/or clinical suspicion for involved deep lymph nodes underwent SGD. SGD was performed via a transverse inguinal incision and involved complete dissection of lymph nodes from the inguinofemoral content to the apex of the femoral triangle where the long saphenous vein joins the femoral vein. Sartorius muscle transposition to cover and protect femoral vessels was selectively performed when adjuvant radiotherapy was to be expected and/or skin was at risk. When CGD was performed, an additional incision was made approximately $3-5 \mathrm{~cm}$ above the line of the inguinal ligament. CGD included dissection of the inguinofemoral and external iliac nodes up to the common iliac artery (if necessary up to the aortic bifurcation) and dissection of the obturator nodes.

\section{Statistics}

Disease-free survival (DFS) was calculated from operation date of lymph node dissection to date of first recurrence at any site. Overall survival (OS) was calculated from operation date of lymph node dissection to date of death due to any cause.

Different statistical methods were applied as appropriate. The chi-square test, Fisher's exact test, and Mann-Whitney test were applied to investigate differences in clinicopathological features, the predictive value of the number of involved superficial nodes for deep lymph node involvement, postoperative morbidities, and regional recurrence patterns in CGD and SGD patients. The log-rank test and the Kaplan-Meier method were assessed for survival analysis and the search for prognostic factors in CGD patients, SGD patients, and the total group of patients. All calculations were performed using STATA version 10.1 and 11.1 (StataCorp LP, College Station, TX, USA).

\section{RESULTS}

\section{CGD Versus $S G D$}

This study included 121 patients (70 women and 51 men) who underwent therapeutic combined superficial and deep dissection (CGD) and 48 patients (32 women and 16 men) who underwent therapeutic superficial dissection (SGD) for palpable melanoma metastases to the groin. Surgeries were performed between 1991 and 2009 at the Erasmus University Medical Center - Daniel den Hoed Cancer Center, Rotterdam, The Netherlands. Median follow-up time was 20 months for all patients and 45 months for all survivors (both, range 1-202 months). Median age at time of surgery was 54 (range 21-87) years. CGD patients had significantly more patients with large superficial nodes than SGD patients ( $p=0.002)$, more harvested superficial lymph nodes $(p<0.001)$, and lower superficial lymph node ratio $(p=0.0004)$ (Table 1$)$.

\section{Preoperative Diagnosis}

Patients were clinically diagnosed by computed tomography (CT), fine-needle aspiration cytology (FNAC), and/or ultrasound. All SGD patients were diagnosed with superficial lymph node involvement only. Of all CGD patients, 24 (19.8\%) were diagnosed with superficial and deep lymph node involvement and 97 (80.2\%) were diagnosed with only superficial lymph node involvement. 
TABLE 1 Clinicopathological factors
$I Q R$ interquartile range

${ }^{a} p$-Values calculated with the Fisher exact test, chi-square test, and Mann-Whitney test

\begin{tabular}{|c|c|c|c|}
\hline & $\begin{array}{l}\text { Combined deep and } \\
\text { superficial groin } \\
\text { dissections } \\
(n=121), n(\%)\end{array}$ & $\begin{array}{l}\text { Superficial groin } \\
\text { dissections } \\
(n=48), n(\%)\end{array}$ & $p$-Value ${ }^{\mathrm{a}}$ \\
\hline \multicolumn{4}{|l|}{ Gender } \\
\hline Female & $70(57.9)$ & $32(66.7)$ & \multirow[t]{2}{*}{0.303} \\
\hline Male & $51(42.2)$ & $16(33.3)$ & \\
\hline \multicolumn{4}{|l|}{ Age (years) } \\
\hline$\leq 50$ & $47(38.8)$ & $11(22.9)$ & \multirow[t]{2}{*}{0.072} \\
\hline$>50$ & $74(61.2)$ & $37(77.1)$ & \\
\hline \multicolumn{4}{|l|}{ Site of primary } \\
\hline Leg & $78(78.8)$ & $37(92.5)$ & \multirow[t]{3}{*}{0.080} \\
\hline Trunk & $21(21.2)$ & $3(7.5)$ & \\
\hline Missing & 22 & 8 & \\
\hline \multicolumn{4}{|l|}{ Breslow thickness (mm) } \\
\hline$\leq 2.00$ & $52(57.1)$ & $14(38.9)$ & \multirow[t]{4}{*}{0.099} \\
\hline $2.01-\leq 4.00$ & $23(25.3)$ & $10(27.8)$ & \\
\hline$>4.00$ & $16(17.6)$ & $12(33.3)$ & \\
\hline Missing & 30 & 12 & \\
\hline \multicolumn{4}{|l|}{ Clark level } \\
\hline II-III & $26(32.1)$ & $9(30)$ & \multirow[t]{4}{*}{0.907} \\
\hline IV & $48(59.3)$ & $19(63.3)$ & \\
\hline $\mathrm{V}$ & $7(8.6)$ & $2(6.7)$ & \\
\hline Missing & 40 & 18 & \\
\hline \multicolumn{4}{|l|}{ Ulceration } \\
\hline Absent & $89(73.6)$ & $34(70.8)$ & \multirow[t]{2}{*}{0.706} \\
\hline Present & $32(26.5)$ & $14(29.2)$ & \\
\hline \multicolumn{4}{|l|}{ Extranodal invasion } \\
\hline Absent & $33(48.5)$ & $14(51.9)$ & \multirow[t]{3}{*}{0.823} \\
\hline Present & $35(51.5)$ & $13(48.2)$ & \\
\hline Missing & 53 & 21 & \\
\hline \multicolumn{4}{|l|}{ Largest diameter of positive superficial node $(\mathrm{cm})$} \\
\hline$<3$ & $21(29.2)$ & $11(50.0)$ & \multirow[t]{3}{*}{0.002} \\
\hline$\geq 3$ & $51(70.8)$ & $11(50.0)$ & \\
\hline Missing & 50 & 26 & \\
\hline \multicolumn{4}{|l|}{ No. positive superficial nodes } \\
\hline 1 & $57(47.1)$ & $26(54.2)$ & \multirow[t]{3}{*}{0.553} \\
\hline $2-3$ & $35(28.9)$ & $14(29.2)$ & \\
\hline$>3$ & $29(24.0)$ & $8(16.7)$ & \\
\hline No. harvested superficial nodes, median (IQR) & $15(12-22)$ & $8(5-14)$ & $<0.001$ \\
\hline \multicolumn{4}{|l|}{ Superficial lymph node ratio $(\%)$} \\
\hline Median (IQR) & $11(6-25)$ & $20(10-50)$ & 0.0004 \\
\hline$\leq 10$ & $54(45.4)$ & $12(25.0)$ & \multirow[t]{4}{*}{0.035} \\
\hline $10-\leq 25$ & $37(31.1)$ & $18(37.5)$ & \\
\hline$>25$ & $28(23.5)$ & $18(37.5)$ & \\
\hline Missing & 2 & 0 & \\
\hline \multicolumn{4}{|l|}{ Positive deep lymph nodes } \\
\hline Absent & $91(75.2)$ & $48(100.0)$ & \multirow[t]{2}{*}{-} \\
\hline Present & $30(24.8)$ & $0(0.0)$ & \\
\hline Adjuvant radiotherapy & $11(9.1)$ & $5(10.4)$ & 0.776 \\
\hline
\end{tabular}


Preoperative CT scans could be retrieved in 61 of 121 CGD patients. Of the 61 radiographically evaluated CGD patients, $44(62.1 \%)$ were diagnosed with only superficial lymph node involvement, of which 40 were histologically confirmed by the pathologist (negative predictive value for pelvic involvement 91\%). Positive predictive value for pelvic metastases was $59 \%$, sensitivity was $71 \%$, and specificity was $85 \%$ (Table 2).

\section{Postoperative Morbidity}

Median hospital stay was 6 (range 3-27) days in patients with CGD and 6 (range 2-32) days in patients with SGD. There were no statistically significant differences in postoperative morbidities between CGD and CGD patients (all $p>0.05)$, although there was a trend towards more chronic lymphedema in the CGD group (25.6\% versus $14.6 \%$, $p=0.154)$ (Table 3).

TABLE 2 CT accuracy for pelvic lymph node involvement in CGD patients

\begin{tabular}{lrcc}
\hline & CT pelvic + & CT pelvic - & Total \\
\hline Histology pelvic + & 10 & 4 & 14 \\
Histology pelvic - & 7 & 40 & 47 \\
Total & 17 & 44 & 61
\end{tabular}

Sensitivity $=10 / 14=71.4 \%$

Specificity $=40 / 47=85.1 \%$

Positive predictive value $=10 / 17=58.8 \%$

Negative predictive value $=40 / 44=90.9 \%$

$C T$ computed tomography, $C G D$ combined deep and superficial groin dissection

\section{Recurrence}

There was no statistical difference in disease-free survival time or time to regional relapse between SGD and CGD patients, with overall recurrence rate of $73 \%(90 / 121)$ and $74 \%$ (35/48), respectively. At time of last follow-up, 81 of 121 patients $(67 \%)$ in the CGD group and 31 of 48 patients $(65 \%)$ in the SGD group were dead. Regional recurrence rates were more common in SGD than in CGD patients, i.e., $21 \%$ and $16 \%(p=0.498)$, and pelvic recurrence rates were $10 \%$ in both groups of patients $(p=1.000)$. Median time to first recurrence was 7.6 (range 1-96) months for CGD patients and 6.0 (range 1-42) months for SGD patients $(p=0.677)$ (Table 3$)$.

\section{Survival Analysis}

Disease-free (DFS) and overall survival (OS) in CGD patients were not better than in SGD patients $(p=0.722$ and $p=0.647$, respectively) (Fig. 1c, d). Comparison of DFS and OS of CGD patients who only had superficial nodes involved with SGD patients also showed no significant difference ( $p=0.421$ and $p=0.217$, respectively).

Five-year estimated DFS and OS rates for patients who underwent SGD were $15.7 \%$ and $28.7 \%$, respectively. Five-year estimated DFS and OS rates for patients who underwent CGD were $18.3 \%$ and $33.0 \%$, respectively (Fig. 1c, d).

On univariate analysis of prognostic factors in the total number of patients $(n=169)$, the number of positive superficial nodes $(1,2-3, \geq 4)$ was a significant prognostic factor for DFS [ $\geq 4$ nodes only; hazard ratio $(\mathrm{HR})=1.85$; 95\% confidence interval (CI) $1.21-2.84 ; p=0.005]$ and OS $\quad(\mathrm{HR}=1.60 ; 95 \%$ CI $1.03-2.51 ; p=0.038$ and

TABLE 3 Postoperative morbidity and regional recurrence rates

\begin{tabular}{|c|c|c|c|}
\hline Type of morbidity & $\begin{array}{l}\text { Combined deep and superficial } \\
\text { groin dissections }(n=121), n(\%)\end{array}$ & $\begin{array}{l}\text { Superficial groin } \\
\text { dissections }(n=48), n(\%)\end{array}$ & $p$-Value ${ }^{\mathrm{a}}$ \\
\hline Overall & $77(63.6)$ & $24(50.0)$ & 0.119 \\
\hline Short term ${ }^{\mathrm{b}}$ & 60 (49.6) & $19(39.6)$ & 0.305 \\
\hline Long term ${ }^{c}$ & $32(26.5)$ & $8(16.7)$ & 0.229 \\
\hline Wound infection and/or necrosis & $30(24.8)$ & $13(27.1)$ & 0.845 \\
\hline Chronic lymphedema & $31(25.6)$ & $7(14.6)$ & 0.154 \\
\hline \multicolumn{4}{|l|}{ Type of recurrence } \\
\hline Median time to recurrence (months) & 7.6 & 6.0 & 0.677 \\
\hline Regional superficial and deep lymph node recurrence & $19(15.7)$ & $10(20.8)$ & 0.498 \\
\hline Of which: Pelvic lymph node recurrence & $12(9.9)$ & $5(10.4)$ & 1.000 \\
\hline
\end{tabular}

${ }^{a} p$-Values calculated with the Mann-Whitney test, Fisher's exact test, and chi-square test

b Short-term morbidities include wound infection and/or necrosis, seroma, postoperative bleeding, urinary tract infection, pulmonary embolism or thrombosis, and transient nerve damage

${ }^{c}$ Long-term morbidities include chronic lymphedema, urinary tract damage, permanent nerve damage, and loss of function 

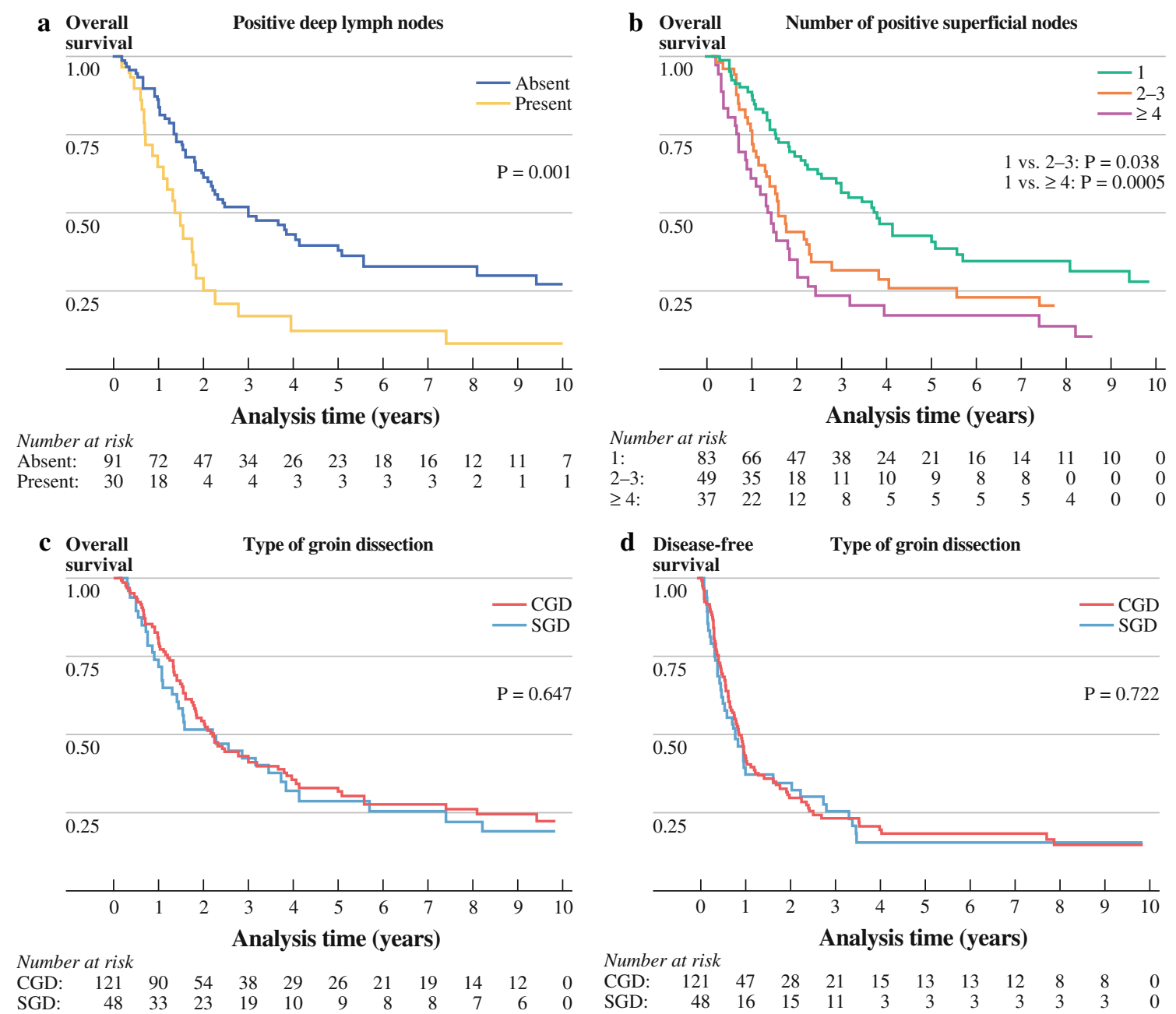

$\begin{array}{lrrrrrrrrrrr}\text { Number at risk } & & & & & & & & \\ \text { CGD: } & 121 & 47 & 28 & 21 & 15 & 13 & 13 & 12 & 8 & 8 & 0 \\ \text { SGD: } & 48 & 16 & 15 & 11 & 3 & 3 & 3 & 3 & 3 & 3 & 0\end{array}$

FIG. 1 Overall survival by (a) positive deep lymph nodes in CGD patients, (b) number of positive superficial nodes in all patients, and (c) type of groin dissection. $\mathbf{d}$ Disease-free survival by type of groin dissection

$\mathrm{HR}=2.36 ; 95 \%$ CI $1.50-3.71 ; p=0.0005)$ as well as superficial lymph node ratio for DFS (HR $=2.33 ; 95 \% \mathrm{CI}$ $1.25-4.34 ; p=0.008)$ and $\mathrm{OS}(\mathrm{HR}=3.16 ; 95 \% \mathrm{CI}$ $1.68-5.94 ; p<0.001)$. Presence of involved deep lymph nodes was a prognostic factor for OS (HR $=1.95 ; 95 \% \mathrm{CI}$ $1.24-3.07 ; p=0.004)$.

On univariate analysis of prognostic factors in SGD patients only, the largest diameter of the positive lymph node was significant for OS $(\mathrm{HR}=3.10 ; 95 \%$ CI $1.07-8.98 ; p=0.037$ ), while analysis in CGD patients revealed superficial lymph node ratio, more than three positive superficial nodes, as well as presence of involved deep lymph nodes as poor prognostic factors for OS $(\mathrm{HR}=5.90,95 \%$ CI 2.21-15.76, $p<0.001 ; \mathrm{HR}=2.29$, 95\% CI 1.34-3.91, $p=0.002$; and $\mathrm{HR}=2.25,95 \% \mathrm{CI}$ $1.38-3.66, p=0.001$, respectively) and DFS $(\mathrm{HR}=4.64$, 95\% CI $1.70-12.65, p=0.003 ; \mathrm{HR}=1.96,95 \%$ CI $1.19-3.22, p=0.008$; and $\mathrm{HR}=1.61,95 \%$ CI $1.02-2.55$, $p=0.041$, respectively) (Table 4 ).
Five-year estimated DFS and OS rates for positive deep lymph nodes were $9.1 \%$ and $12.5 \%$, respectively, compared with 5-year estimated DFS and OS rates for positive superficial lymph nodes only in CGD patients of $21.5 \%$ and $39.7 \%$ (Fig. 1a). Five-year estimated DFS rates for the number of positive superficial lymph nodes was $23.7 \%$ for $1,12.0 \%$ for $2-3$, and $11.2 \%$ for $\geq 4$ involved nodes. Fiveyear estimated OS rates for the number of positive superficial lymph nodes was $42.6 \%$ for $1,25.8 \%$ for $2-3$, and $17.1 \%$ for $\geq 4$ involved nodes (Fig. 1b).

\section{DISCUSSION}

Survival in patients with palpable metastatic melanoma to the groin is poor. In the literature, estimated 5-year overall survival (OS) rates vary from $20 \%$ to $40 \% .^{8,11,13}$ In our series of 169 patients with palpable nodes in the groin, 5-year estimated OS rates were 33\% for CGD and 29\% for SGD. Also 5-year DFS rates were virtually identical, i.e., 
TABLE 4 Cox regression univariate analysis of overall and disease-free survival for prognostic factors in CGD and SGD patients, and the total group of patients

\begin{tabular}{|c|c|c|c|c|c|c|c|c|c|}
\hline & \multirow{2}{*}{\multicolumn{3}{|c|}{$\begin{array}{l}\text { Combined deep and superficial groin dissections } \\
(n=121) \\
\text { Disease-free survival }^{\mathrm{a}}\end{array}$}} & \multicolumn{3}{|c|}{$\begin{array}{l}\text { Superficial groin dissections } \\
(n=48)\end{array}$} & \multicolumn{3}{|c|}{ All dissections $(n=169)$} \\
\hline & & & & & & & & & \\
\hline & HR & $95 \% \mathrm{CI}$ & $p$ & HR & $95 \% \mathrm{CI}$ & $p$ & HR & $95 \% \mathrm{CI}$ & $p$ \\
\hline \multicolumn{10}{|c|}{ Largest diameter of superficial node $(\mathrm{cm})$} \\
\hline$<3$ & 1 & & & 1 & & & 1 & & \\
\hline$\geq 3$ & 1.69 & $0.90-3.17$ & 0.100 & 2.48 & $0.91-6.80$ & 0.077 & 1.82 & $1.08-3.07$ & 0.024 \\
\hline \multicolumn{10}{|l|}{ No. of positive superficial nodes } \\
\hline 1 & 1 & & & 1 & & & 1 & & \\
\hline $2-3$ & 1.53 & $0.93-2.51$ & 0.092 & 1.29 & $0.63-2.66$ & 0.494 & 1.40 & $0.93-2.11$ & 0.103 \\
\hline$\geq 4$ & 1.96 & $1.19-3.22$ & 0.008 & 1.85 & $0.77-4.41$ & 0.167 & 1.85 & $1.21-2.84$ & 0.005 \\
\hline Superficial lymph node ratio & 4.64 & $1.70-12.65$ & 0.003 & 1.64 & $0.66-4.08$ & 0.283 & 2.33 & $1.25-4.34$ & 0.008 \\
\hline \multicolumn{10}{|l|}{ Positive deep nodes } \\
\hline Absent & 1 & & & & & & 1 & & \\
\hline Present & 1.61 & $1.02-2.55$ & 0.041 & & & $\mathrm{~N} / \mathrm{A}^{\mathrm{b}}$ & 1.48 & $0.96-2.28$ & 0.075 \\
\hline \multicolumn{10}{|l|}{ Overall survival $^{\mathrm{a}}$} \\
\hline \multicolumn{10}{|c|}{ Largest diameter of superficial node $(\mathrm{cm})$} \\
\hline$<3$ & 1 & & & 1 & & & 1 & & \\
\hline$\geq 3$ & 1.43 & $0.74-2.77$ & 0.292 & 3.10 & $1.07-8.98$ & 0.037 & 1.72 & $0.99-3.00$ & 0.055 \\
\hline \multicolumn{10}{|l|}{ No. of positive superficial nodes } \\
\hline 1 & 1 & & & 1 & & & 1 & & \\
\hline $2-3$ & 1.66 & $0.96-2.87$ & 0.071 & 1.48 & $0.69-3.17$ & 0.316 & 1.60 & $1.03-2.51$ & 0.038 \\
\hline$\geq 4$ & 2.29 & $1.34-3.91$ & 0.002 & 2.44 & $0.99-6.01$ & 0.052 & 2.36 & $1.50-3.71$ & 0.0005 \\
\hline Superficial lymph node ratio & 5.90 & $2.21-15.76$ & $<0.001$ & 2.27 & $0.88-5.88$ & 0.091 & 3.16 & $1.68-5.94$ & $<0.001$ \\
\hline \multicolumn{10}{|l|}{ Positive deep nodes } \\
\hline Absent & 1 & & & & & & 1 & & \\
\hline Present & 2.25 & $1.38-3.66$ & 0.001 & & & $\mathrm{~N} / \mathrm{A}^{\mathrm{b}}$ & 1.95 & $1.24-3.07$ & 0.004 \\
\hline
\end{tabular}

N/A not applicable, $C G D$ combined deep and superficial groin dissection, $S G D$ superficial groin dissections

a The following variables did not have any prognostic significance in all groups: gender, age, site of primary, Breslow thickness, Clark level, ulceration, and extranodal invasion

b Variable not assessable due to no presence of positive deep nodes

$18 \%$ for CGD and $16 \%$ for SGD. Patients with CGD with positive deep nodes have the poorest prognosis, with OS ranging from $6 \%$ to $34 \%$ in the literature. . $^{7,8,10,14-16}$ (Table 5). In our institute, patients with CGD and positive deep nodes have estimated 5-year OS and DFS rates of $12 \%$ and $9 \%$. In contrast, for CGD patients without deep nodal involvement, we observed $40 \%$ and $22 \%$, respectively.

There were differences between the CGD and SGD patients. CGD patients had a significantly larger size of involved superficial lymph nodes than SGD patients (Table 1). Moreover, 25\% of CGD patients had involved deep lymph nodes, while there was no suspicion and no diagnosis of deep nodal involvement in SGD patients. CGD patients had unfavorable preoperative prognosis, which is apparent since selection for extent of surgery was based on comorbidities and the suspicion of involvement of deep lymph nodes. However, during CGD, more superficial nodes were harvested and the number of positive superficial nodes was not different, resulting in a significant lower superficial lymph node ratio (Table 1). Lower superficial lymph node ratio is a good prognostic factor for survival. ${ }^{17-19}$ Based on surgery only, CGD patients were expected to have favorable prognosis. In this study, the outcome of CGD patients was virtually identical to that of SGD patients (Fig. 1c, d). Even comparison of patients with superficial involved nodes only showed no difference, indicating that the extent of groin surgery does not influence outcome (data not shown; $p=0.217$ ). Also in other studies, it has been demonstrated that extent of groin surgery, regardless of presence or absence of deep lymph node involvement in CGD patients, has no effect on survival. ${ }^{8,10,16}$ 
TABLE 5 Overview of literature describing survival rates in patients with positive deep nodes to the groin diagnosed after therapeutic combined deep and superficial lymph node dissection only

\begin{tabular}{|c|c|c|c|c|c|c|}
\hline Institute & Reference & Year & Study period & $\begin{array}{l}\text { Median } \\
\text { follow-up } \\
\text { (months) }\end{array}$ & $\begin{array}{l}\text { No. of patients with } \\
\text { positive pelvic nodes } \\
\text { (\% of total) }\end{array}$ & 5 -Year OS $(\%)$ \\
\hline NCI/ALH & $\begin{array}{l}\text { Jonk }^{\text {a, } 27} \\
\text { Strobbe }^{7}\end{array}$ & 1999 & 1961-1995 & 18 & $71(20)$ & 24 \\
\hline UCLA & Finck $^{14}$ & 1982 & 1970-1980 & 23 & $24(29)$ & 17 \\
\hline \multirow[t]{2}{*}{ MSKCC } & Coit $^{10}$ & 1989 & 1974-1984 & $86^{\mathrm{b}}$ & $10(7)$ & 6 \\
\hline & Mann $^{8}$ & 1999 & 1985-1994 & 40 & $21(19)$ & \pm 35 \\
\hline RPCI & Karakousis c, 2,3,15,28 & 1996 & 1977-1993 & \pm 46 & 48 (NR) & 34 \\
\hline UE & Meyer $^{13}$ & 2002 & 1978-1997 & 20 & $23(31)$ & 21 \\
\hline MLUHW & Kretschmer $^{16}$ & 2001 & 1983-1994 & $68^{\mathrm{b}}$ & $24(35)$ & 6 \\
\hline $\mathrm{RMH}$ & Hughes $^{11}$ & 2000 & 1984-1998 & 19 & $29(40)$ & 19 \\
\hline MDACC & Badgwell $^{29}$ & 2007 & 1990-2001 & 90 & $55(51)^{\mathrm{d}}$ & $42^{\mathrm{d}}$ \\
\hline DDHCC & Recent study & 2011 & 1991-2009 & 20 & $30(25)$ & 12 \\
\hline
\end{tabular}

$O S$ overall survival; $N R$ not reported; $N C I / A L H$ Netherlands Cancer Institute/Antoni van Leeuwenhoek Hospital, Amsterdam, The Netherlands; UCLA University of California, Los Angeles, CA; MSKCC Memorial Sloan-Kettering Cancer Center, New York, NY; RPCI Roswell Park Cancer Institute, Buffalo, NY; UE University of Erlangen, Erlangen, Germany; MLU $H W$ Martin-Luther-Universitat Halle-Wittenberg, Halle, Germany; RMH Melanoma and Sarcoma Unit, Royal Marsden Hospital, London, UK; MDACC M.D. Anderson Cancer Center, Houston, TX; DDHCC Erasmus Medical Center - Daniel Den Hoed Cancer Center, Rotterdam, The Netherlands

${ }^{\text {a }}$ The 23 patients (5-year overall survival 32\%) reported by Jonk et al. in 1989 are included in the study performed by Strobbe et al

b The median survival shown is for patients who survived only. Median follow-up for the entire group is not reported

c The patients described in the three earlier reports (1986 and two reports in 1994) of Karakousis et al. are included in the 1996 study

d The patients in this study underwent any type of lymph node dissection and not only therapeutic lymph node dissections

Preoperative CT was performed in 61 of 121 patients who underwent CGD. Positive predictive value (PPV) of CT scan was only $59 \%$ in our experience, whilst the negative predictive value (NPV) was fairly good at $91 \%$. Sensitivity was $71 \%$ and specificity $85 \%$ in our group of patients (Table 2). Allen et al. found different results with PPV of $100 \%$, NPV of $86 \%$, specificity of $100 \%$, and sensitivity of $60 \%$, stating that CT scan was not reliable as a tool for preoperatively assessing pelvic lymph node involvement. ${ }^{4}$ However, both studies show that a CT-based decision on whether or not to perform CGD could be correctly made in 9 out of 10 patients. Thus, CT scan may be used as a tool in the decision on whether or not to remove deep lymph nodes.

Morbidity rates in the present study are divided into short- and long-term morbidities. Underestimation of events in the morbidity data could have occurred due to the retrospective gathering of data from medical records. However, comparison of the two groups of patients in this study remains valid since this presumed underestimation arose in both groups. Neither short- nor long-term morbidities were significantly lower in SGD than in CGD patients, being $39.6 \%$ versus $49.6 \%(p=0.305)$ and $16.7 \%$ versus $26.5 \%(p=0.229)$, respectively (Table 3$)$. The most debilitating morbidity is chronic lymphedema, which is difficult to define. Some authors have used measurements to define this, whilst others have opted to define chronic lymphedema as edema requiring intervention. Also debated is the minimum period of edema to define it as chronic, which we did when moderate or severe swelling was present (more than) 6 weeks after surgery and required treatment. In any case, it is a widely feared and unpleasant complication. ${ }^{20-22}$ There was a trend towards increased chronic lymphedema in patients after CGD $(25.6 \%)$ than in patients after SGD (14.6\%), yet this difference was not statistically significant $(p=0.154)$. This difference was not the result of an imbalance of additional radiotherapy to the groin, as $10 \%$ of SGD patients received radiotherapy versus $9 \%$ of CGD patients $(p=0.776)$. The assessment of one large or two small incisions for CGD has no influence on the rate of lymphedema as well. ${ }^{23}$ Other reports also indicate that lymphedema rates after CGD (range 23-55\%) are greater than after SGD (range 7-29\%), albeit not always statistically significantly so. ${ }^{8,11,20,22}$ Faries et al. recently reported the difference in lymphedema rates between immediate and delayed lymph node dissection. In these data of the Multicenter Selective Lymphadenectomy Trial (MSLT) I, lymphedema rates for SGD patients were $21.4 \%$ when undergoing immediate dissection and $22.6 \%$ when undergoing delayed dissection $(p=0.9)$, while CGD patients had higher lymphedema rates of $36.4 \%$ for immediate dissection and $34.2 \%$ for 
delayed dissection $(p=0.89){ }^{22}$ Unfortunately, $p$-values for the difference in lymphedema between SGD and CGD patients were not provided. ${ }^{22}$

Regarding locoregional control in the ilioinguinal region, we found no differences between CGD and SGD, as regional recurrence rates were similar (Table 3). The frequency of pelvic recurrences was equal in both groups. Possible causes for this counterintuitive observation could be the small sample size, patient selection, i.e., SGD patients having occult pelvic disease at time of surgery, and/or the overall worse prognosis of both groups of patients compared with literature. Patients might die of distant visceral metastases before pelvic recurrence has developed. Our results are in line with other reports in the literature. Coit et al. reported similar nodal recurrence rates for SGD and CGD patients, while Singletary et al. reported relatively more nodal recurrence in SGD patients, but attributed that to the extent of tumor burden rather than the extent of surgery. ${ }^{10,24}$

Our group of patients has worse survival compared with literature; for example, Balch et al. reported 5-year OS rates of $50 \%$ for $\mathrm{N} 1 \mathrm{~b}, 45 \%$ for $\mathrm{N} 2 \mathrm{~b}$, and $40 \%$ for $\mathrm{N} 3$ patients in the 2009 AJCC melanoma staging system analysis. ${ }^{25}$ Patients from our center showed (in the same order) 5 -year OS rates of $43 \%, 26 \%$, and $17 \%$. Because of our relatively small study population compared with the enormous AJCC databases of $>30,000$ patients, a single event will have a greater impact on the estimate survival rates in Kaplan-Meier analysis, because the number at risk is smaller. Due to our relatively short median follow-up, we underestimate our long-term survival. Moreover, all patients were operated at the Erasmus University Medical Center - Daniel den Hoed Cancer Center, a tertiary center in The Netherlands for such cases. This implies that a negative selection bias is most likely. More advanced cases might lead to worse survival.

As well as the superficial lymph node ratio, the number of positive superficial nodes was a consistent prognostic variable for OS and DFS (Table 4). This is consistent with the outcome after analysis of stage III melanoma patients by the AJCC. ${ }^{25,26}$ With an increasing number of positive superficial lymph nodes, the chance of involvement of the deep lymph nodes increased. No patients with 1 involved superficial node showed additional positivity in the pelvic area, while this applied to $32 \%$ of patients with 2-3 involved superficial lymph nodes and to $66 \%$ of patients with $\geq 4$ involved superficial lymph nodes. A decision on the extent of surgery might be made based on the number of involved superficial lymph nodes. A scenario based on our results of preoperative CT scan and the number of involved superficial lymph nodes could be considered. When preoperative $\mathrm{CT}$ is negative for involvement in the pelvic region and only one superficial lymph node is involved, SGD might be performed. When, after pathological analyses of the SGD specimen, more than one involved superficial lymph node is found, additional dissection of the pelvic region could be considered. CGD is performed in case of suspicion of multiple positive superficial lymph nodes and/or positive deep lymph nodes.

We acknowledge that this study is retrospective and has short follow-up time. We selected patients who underwent only therapeutic groin dissection for palpable disease and excluded patients who underwent elective lymph node dissection or sentinel node biopsy. The consequence was that our clean cohort of melanoma patients underwent surgery in a period of time (1991-2009) with evolving practice and imaging. Before applying this scenario in clinical practice, results similar to those of the present study should be reported by other retrospective studies or a randomized controlled trial.

In conclusion, the poor outcome in melanoma patients with palpable nodal disease in the groin after CGD was equal after SGD in our series and in many other reports in the literature. Patients without overt iliac nodes on CT might safely undergo SGD and be spared the greater morbidity of CGD. CGD might be reserved for patients with multiple positive nodes in the SGD and/or positive nodes on CT scan. A prospective randomized controlled trial is the only study that could overcome the classical drawbacks of this and other retrospective studies.

Conflict of interest We have no conflicts of interest to report.

Open Access This article is distributed under the terms of the Creative Commons Attribution Noncommercial License which permits any noncommercial use, distribution, and reproduction in any medium, provided the original author(s) and source are credited.

\section{REFERENCES}

1. Balch CM, Ross MI. Melanoma patients with iliac nodal metastases can be cured. Ann Surg Oncol. 1999;6(3):230-1.

2. Karakousis CP, Driscoll DL. Groin dissection in malignant melanoma. Br J Surg. 1994;81(12):1771-4.

3. Karakousis CP, Emrich LJ, Rao U. Groin dissection in malignant melanoma. Am J Surg. 1986;152(5):491-5.

4. Allan CP, Hayes AJ, Thomas JM. Ilioinguinal lymph node dissection for palpable metastatic melanoma to the groin. ANZ J Surg. 2008;78(11):982-6.

5. Mack LA, McKinnon JG. Controversies in the management of metastatic melanoma to regional lymphatic basins. J Surg Oncol. 2004;86(4):189-99.

6. Sterne GD, Murray DS, Grimley RP. Ilioinguinal block dissection for malignant melanoma. Br J Surg. 1995;82(8):1057-9.

7. Strobbe LJ, Jonk A, Hart AA, et al. Positive iliac and obturator nodes in melanoma: survival and prognostic factors. Ann Surg Oncol. 1999;6(3):255-62.

8. Mann GB, Coit DG. Does the extent of operation influence the prognosis in patients with melanoma metastatic to inguinal nodes? Ann Surg Oncol. 1999;6(3):263-71. 
9. McCarthy JG, Haagensen CD, Herter FP. The role of groin dissection in the management of melanoma of the lower extremity. Ann Surg. 1974;179(2):156-9.

10. Coit DG, Brennan MF. Extent of lymph node dissection in melanoma of the trunk or lower extremity. Arch Surg. 1989; 124(2):162-6.

11. Hughes TM, A'Hern RP, Thomas JM. Prognosis and surgical management of patients with palpable inguinal lymph node metastases from melanoma. Br J Surg. 2000;87(7):892-901.

12. Hughes TM, Thomas JM. Combined inguinal and pelvic lymph node dissection for stage III melanoma. Br J Surg. 1999;86(12): 1493-8.

13. Meyer T, Merkel S, Gohl J, Hohenberger W. Lymph node dissection for clinically evident lymph node metastases of malignant melanoma. Eur J Surg Oncol. 2002;28(4):424-30.

14. Finck SJ, Giuliano AE, Mann BD, Morton DL. Results of ilioinguinal dissection for stage II melanoma. Ann Surg. 1982; 196(2):180-6.

15. Karakousis CP, Driscoll DL. Positive deep nodes in the groin and survival in malignant melanoma. Am J Surg. 1996;171(4):421-2.

16. Kretschmer L, Neumann C, Preusser KP, Marsch WC. Superficial inguinal and radical ilioinguinal lymph node dissection in patients with palpable melanoma metastases to the groin-an analysis of survival and local recurrence. Acta Oncol. 2001;40(1): $72-8$.

17. Xing Y, Badgwell BD, Ross MI, et al. Lymph node ratio predicts disease-specific survival in melanoma patients. Cancer. 2009;115(11):2505-13.

18. Spillane AJ, Cheung BL, Winstanley J, Thompson JF. Lymph node ratio provides prognostic information in addition to AJCC N stage in patients with melanoma, even if quality of surgery is standardized. Ann Surg. 2011;253(1):109-15.

19. Spillane AJ, Winstanley J, Thompson JF. Lymph node ratio in melanoma: A marker of variation in surgical quality? Cancer. 2009;115(11):2384-7.
20. Spillane AJ, Saw RP, Tucker M, et al. Defining lower limb lymphedema after inguinal or ilio-inguinal dissection in patients with melanoma using classification and regression tree analysis. Ann Surg. 2008;248(2):286-93.

21. Tobin MB, Lacey HJ, Meyer L, Mortimer PS. The psychological morbidity of breast cancer-related arm swelling. Psychological morbidity of lymphoedema. Cancer. 1993;72(11):3248-52.

22. Faries MB, Thompson JF, Cochran A, et al. The impact on morbidity and length of stay of early versus delayed complete lymphadenectomy in melanoma: results of the Multicenter Selective Lymphadenectomy Trial (I). Ann Surg Oncol. 2010; 17(12):3324-9.

23. Spillane AJ, Tucker M, Pasquali S. A pilot study reporting outcomes for melanoma patients of a minimal access ilio-inguinal dissection technique based on two incisions. Ann Surg Oncol. 2011;18(4):970-6.

24. Singletary SE, Shallenberger R, Guinee VF. Surgical management of groin nodal metastases from primary melanoma of the lower extremity. Surg Gynecol Obstet. 1992;174(3):195-200.

25. Balch CM, Gershenwald JE, Soong SJ, et al. Final version of 2009 AJCC melanoma staging and classification. J Clin Oncol. 2009;27(36):6199-206.

26. Balch CM, Gershenwald JE, Soong SJ, et al. Multivariate analysis of prognostic factors among 2,313 patients with stage III melanoma: comparison of nodal micrometastases versus macrometastases. J Clin Oncol. 2010;28(14):2452-9.

27. Jonk A, Kroon BB, Rumke P, et al. Results of radical dissection of the groin in patients with stage II melanoma and histologically proved metastases of the iliac or obturator lymph nodes, or both. Surg Gynecol Obstet. 1988;167(1):28-32.

28. Karakousis CP, Driscoll DL, Rose B, Walsh DL. Groin dissection in malignant melanoma. Ann Surg Oncol. 1994;1(4):271-7.

29. Badgwell B, Xing Y, Gershenwald JE, et al. Pelvic lymph node dissection is beneficial in subsets of patients with node-positive melanoma. Ann Surg Oncol. 2007;14(10):2867-75. 